\title{
Analysis of LTE Wireless Network Planning in Power System
}

\author{
Qing Liu, Tie Guo, Jiwen Yu, Yongchao Luo and Fan Liu
}

State Grid Jiangxi Electric Power Research Institute, Jiangxi Nanchang 33009)

\section{Keywords: LTE; Network planning; Coverage; Link budget}

\begin{abstract}
With the popularity of intelligent terminals and the rapid growth of mobile data services in recent years, the demand for mobile broadband network is greatly increased. LTE system proposed by 3GPP uses new air interface technology and flattened IP network architecture that can provide the peak rate with downlink $100 \mathrm{Mbit} / \mathrm{s}$ and uplink $50 \mathrm{Mbit} / \mathrm{s}$, and therefore becomes the mainstream technology evolution program for effectively reacting to the explosive growth of global mobile data business. Based on the characteristics of LTE technology and the general planning method, this paper designs a wireless plan for the LTE network of a certain regional telecommunication from two respects of coverage planning and capacity planning, and then verifies the planning through simulation and test, so as to resolve the existing problems of network coverage by means of optimization. The paper estimates out the throughput of each user's uplink and downlink by analyzing both business model and traffic model, and then determines the number of users supportable for each district in accordance with the community capacity, and so as to obtain the number of base stations needed from capacity angle by combining the numbers forecasted by market.
\end{abstract}

\section{Introduction}

In order to support the core tasks and objectives of smart grid construction, it is necessary to build a wide coverable and flexible accessible terminal access network not just to rely on optical fiber access network which cannot meet the communication needs of different regions and businesses such as: the business of the core city, the business of the ancient city area, the business of the residential area and the massive smart grid intelligent management business relying on the mobile operation terminal for the reason of its high constructional costs, long constructional period or infeasibility at all. While, the wireless communication network is more suitable for dispersed distributed power terminals, especially for the communication needs of mobile service terminals. In our country, wired method is taken at present to solve the communication problems of the backbone network of the distribution network while the wireless public network is as a supplementation. This way has been exposed out so many disadvantages in the actual construction event. Optical fiber communication or carrier communication as the leading cable communication cannot solve the problem of large numbers and big range of coverage of the medium-low voltage distribution network, while the wireless public network now has low security and limit bandwidth traffic that it has no ability to be used as the main communication system in the power system, so the establishment of wireless specialized network is very necessary. In this paper, certain regions are taken as the examples to introduce the LTE wireless specialized network planning of the power system in a detailed way.

\section{Process for LTE Wireless Network Planning}

Network planning mainly includes wireless network, transmission network and the core network, among which the planning of wireless network is the most important one and also the basis of the planning of the other two. Wireless network planning is mainly parameter configuration planning, transmission network planning is for the choice of link budget and connection mode, and the core network planning is the one for network elements number. LTE network planning process includes: network planning requirements analysis, network scope estimation, site selection, coverage capacity simulation, and wireless parameter planning, etc. LTE network planning process shows in Fig. 1: 


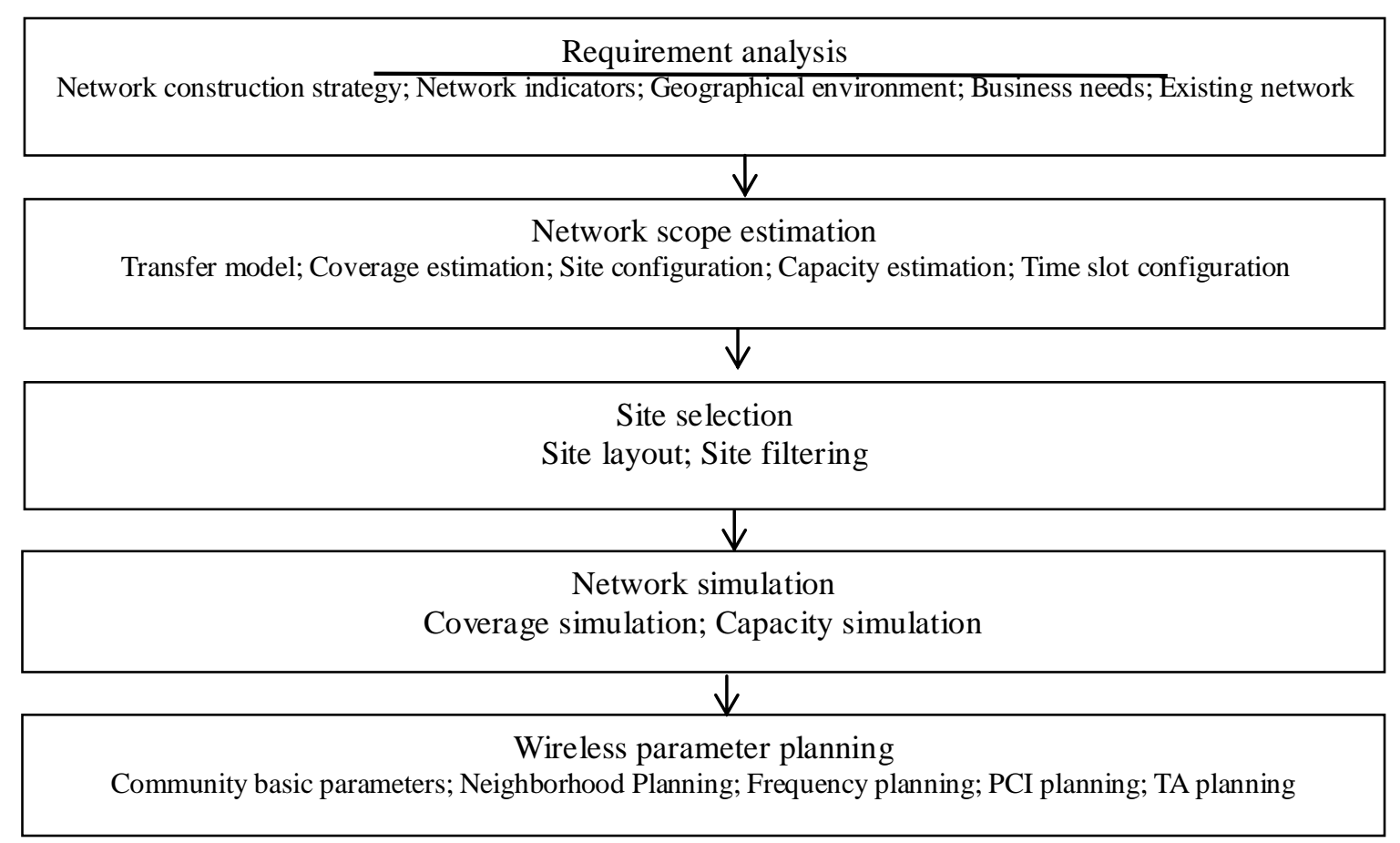

Figure 1. LTE wireless network planning process

At the stage of demand analysis, a corresponding network construction indictor shall be put forward after defining about the network construction, and then accessed business type and information of the base site shall be naileddown.

The network scope estimation mainly includes coverage estimation and capacity estimation, through which the network scope is calculated, and meanwhile through the transfer model and line budget, community coverage radius can be calculated and the numbers of base sites can be nailed down.

Site planning is planned in accordance with the link budget and situations of the site. It is followed by site survey, availability analysis and the final clearance of site's location.

When the site planning completed, it is time to carry out the coverage simulation analysis by using planning simulation software and the analysis steps include: parameter setting, transformation prediction, neighborhood planning, and time slot and frequency configuration, etc. Simulation results can be used to assess whether the planning is reasonable, so that the planning can be adjusted accordingly if unreasonable. After the simulation which carried out by the software, the plan can be done according to specific circumstances.

\section{Construction Goals}

Coverage Goal. Coverage goals of LTE network mainly means: when setting the area coverage index, the current urban area coverage rate of $3 \mathrm{G}$ shall be mainly referenced along with the consideration of the same regional index of competitors, so as to make the proportion of coverage area of the main city be higher than $95 \%$ and the public reference signal received power (RSRP) not less than $-105 \mathrm{~d} \mathrm{Bm}$.

Capacity Goal. The downlink average rate of LTE network in the community can equal to $24 \mathrm{Mbps}$ or higher and the uplink average rate be $10 \mathrm{Mbps}$ or higher in the situation that the neighbor has $50 \%$ load and takes $15 \mathrm{MHz} \times 2$ capacity.

Business Carrying Goal. Providing all-around mobile internet business, LTE consequently becomes the main carrying subject for high-speed mobile internet and at the same time undertakes the CDMA business and also has ability to make cutover with CDMA. 


\section{Link budget based on site planning}

Link budget is an important part of wireless network planning. The link budget calculates the maximum allowable path loss of the air link by accounting for the gain, margin and loss in the link, thus determining the cell coverage range and the sites spacing in conjunction with the transfer model.

Due to the use of OFDM modulation, LTE system can get the user's data rate by a combined effect of the allocated number of PRB and the selected MCS level, so the link budget for the edge rate adjustment is mainly for the balance of the PRB number and MCS selection to achieve a better coverage performance.

A general link budget means to calculate the path loss after setting kinds of gain, loss, and margin data by defining the system parameters and indicators to get uplink and downlink coverage radius of the district, and then take the minimum as the district's radius to calculate its coverage area so as to obtain the needed number of base sites in this region. The specific process is as below Fig. 2:

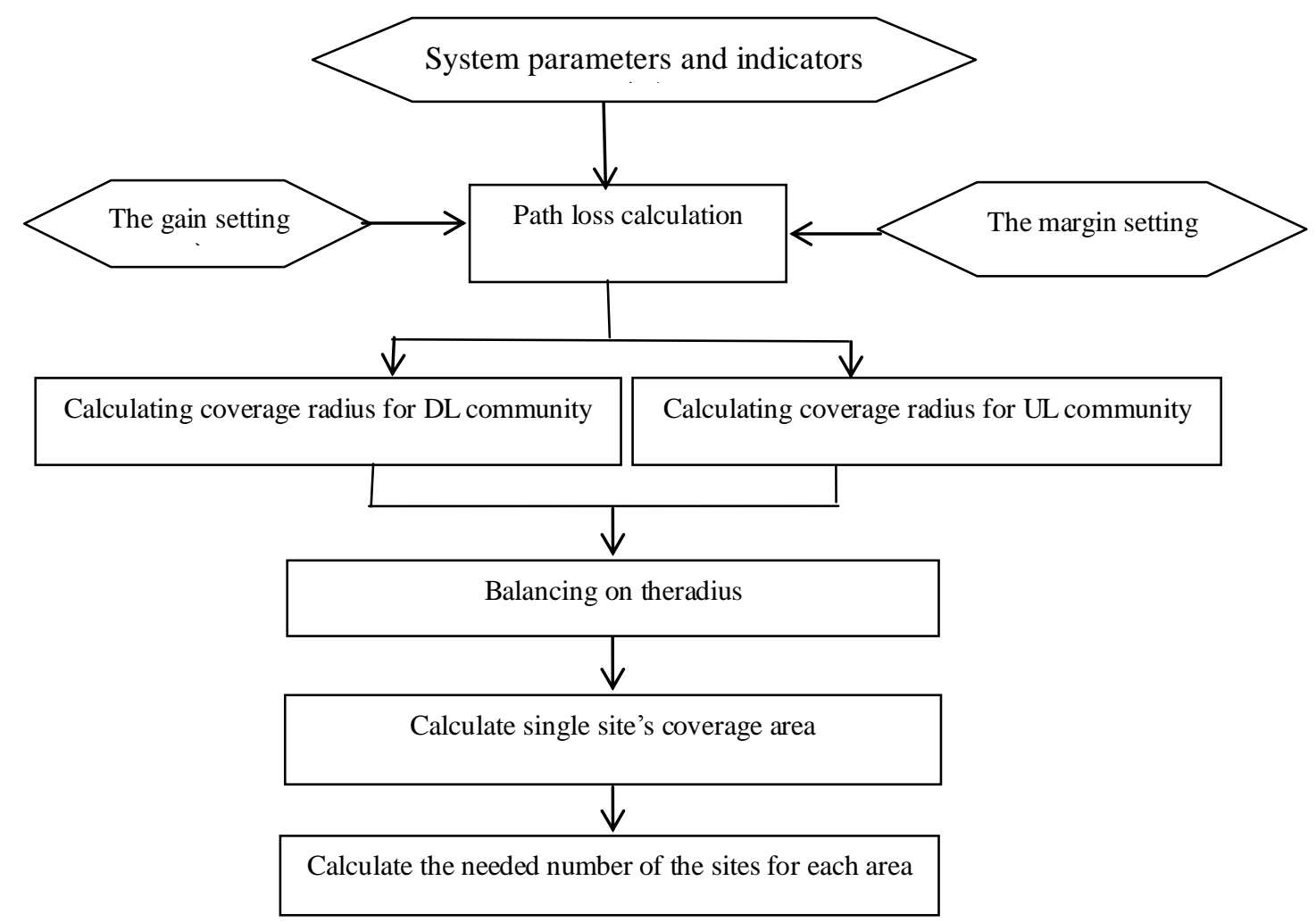

Figure 2. Process map for link budget

In the calculation of the maximum path loss, we generally first calculate the transmitter's EIRP and receiver's receiving sensitivity and then do accumulation of all kinds of gain along with deduction of the loss and finally take the partial project margin into consideration: path loss = transmitter EIRP + gain - loss - project margin - receiver's receiving sensitivity.

According to differences of frequency scenes, there are many transfer models for wireless network and the ones be commonly used are: Okumura-Hata model for $900 \mathrm{MHz}$ microcell base station prediction, COST231-Hata model for $1800 \mathrm{MHz}$ macro cell base site forecast, COST231 Walfish-lkegami Model suitable for 900 to $1800 \mathrm{MHz}$ microcell base site prediction, Keenan-Motley model used in 900 to $1800 \mathrm{MHz}$ indoor environment prediction and the SPM propagation model from Atoll software which is more suitable for LTE systems under dense urban areas of communication environment. The paper makes the planning simulation and calculation in the basis of SPM propagation model.

Here is the SPM standard propagation model: 


$$
P_{R}=P_{T X}-\left(\begin{array}{l}
K_{1}+K_{2} \times \log (d)+K_{3} \times \log \left(H_{T X_{\text {eff }}}\right)+K_{4} \times \text { DiffractionLoss }+K_{5} \times \log (d) \times \log \left(H_{T X_{\text {eff }}}\right) \\
+K_{6} \times H_{R X_{\text {eff }}}+K_{7} \times \log \left(H_{R X_{\text {eff }}}\right)+K_{\text {clutter }} \times f(\text { clutter })+K_{\text {hill.Los }}
\end{array}\right)
$$

The parameters and the meanings of them in this formula are as shown in below Table 1.

Table 1 Explanation on parameters of SPM propagation model

\begin{tabular}{|c|c|}
\hline Parameter & Meaning \\
\hline $\mathrm{P}_{\mathrm{R}}$ & Received power (d Bm) \\
\hline $\mathrm{P}_{\mathrm{TX}}$ & Transmit power (EIRP) (d Bm) \\
\hline $\mathrm{K}_{1}$ & Offset constant (d B) \\
\hline $\mathrm{K}_{2}$ & Product factor of log(d) \\
\hline $\mathrm{d}$ & Product factor of log(HTxeff) \\
\hline $\mathrm{K}_{3}$ & Distance between the receiver and the transmitter (m) \\
\hline $\mathrm{K}_{4}$ & Product factor of diffraction calculation, K4 must be positive \\
\hline Diffraction Loss & Product factor of log(HTxeff)log(d) \\
\hline $\mathrm{K}_{5}$ & Product factor of HRxeff \\
\hline $\mathrm{K}_{6}$ & Product factor of log(RTxeff) \\
\hline $\mathrm{K}_{7}$ & Effective phone antenna height (m) \\
\hline $\mathrm{H}_{\mathrm{RXeff}}$ & Effective phone antenna height (m) \\
\hline $\mathrm{K}_{\mathrm{clutter}}$ & The weighted average loss caused by landforms \\
\hline $\mathrm{f}_{\text {clutter })}$ & Correction factor for mountain area (0 for non-line-of-sight) \\
\hline $\mathrm{K}_{\text {hill, Los }}$ &
\end{tabular}

In this formula, the distance between the receiver and the transmitter can be calculated as d, and then through which the cell radius can be calculated to carry out the coverage area. It is generally assumed that the coverage area of cells of the omnidirectional site is 2.6 times of its radius and is 1.95 times for the one of the three-sector base site. By comparing the area of the needed coverage area and the cell's area, the number of sites of the coverage area in the region can be finally obtained.

\section{Conclusion}

In the basis of the basic principles of LTE network structure and link budget, this paper analyzes the coverage, link budget, parameter setting and simulation system of power system wireless network planning, and proposes a LTE in power system Network planning process, and makes the planning simulation. By network planning simulation software, this papermakes a planning simulation for the power system, and analyzes the result of the simulation through methods of data dealing and comparing analysis to obtain the conclusion that verifies the feasibility of the network planning which can provide effective prediction on signal coverage, save the construction investments and too speed up the progress. Although this paper puts forward the idea of power system wireless network planning, the network construction should take its main duty on network coverage which mainly means to enhance the coverage range and standard to increase the coverage rate, because that the network planning involves a wide range of technologies and each of the practical project has its own particularity, and also because that LTE is in the early stage of network construction.

\section{References}

[1] Y Wang, Z Song and J Bian, et al. Research on MAC Protocol in Wireless Sensor Networks for Electricity Information Collection System[J]. Modern Electric Power, 2013.

[2] X.Y Lin, G.J Tan and Y Zhang, et al. The Application of FTP Remote Upgrade in Electricity Information Collection System[J]. Electrical Measurement \& Instrumentation, 2014. 
[3] H.Q Peng, New Technology of Electricity Information Collection System based on Smart Grid[J]. Information Security \& Technology, 2014.

[4] J.H Xiao, Y.C Wang and C Chen, Design of the Interface between EPON INMS and Electricity Information Collection System[J]. Information Security \& Technology, 2016.

[5] J Li, Q Chen and S.C Yang, The constructive situation and developing direction of the electricity consumption information collection system in China[C]// China International Conference on Electricity Distribution. 2012:1-8.

[6] L.V Zhi-Gang, Analysis of operation difficulties of city grid low-voltage electricity information collection system construction[J]. Power Demand Side Management, 2011.

[7] Z.Wu, The Analysis of the Function of Electricity Information Collection System Based on Wireless Network and Narrowband Power Line Carrier[J]. Wireless Internet Technology, 2015.

[8] H Xiong, Site commissioning experience of broadband electricity utilization information collection

[9] X.B Zhang, W Liu, Study on Electricity Consumer Information Collection System in Distribution Network[J]. Shaanxi Electric Power, 2012.

[10]L Wang, Application of Intelligent Electric Meter in Electricity Utilization Information Collection System of Qinghai Power Grid[J]. Qinghai Electric Power, 2011.

[11] User's Transformer Attribute Determining in the mode of Electricity Information Collection System[J]. Anhui Electric Power, 2013.

[12] J Xiao, J Sun and H Chen, et al. Research on operation environment simulation and detection on electricity information collection terminal[J]. Electrical Measurement \& Instrumentation, 2016. 